\title{
Fractional Filters: An Optimization Approach
}

\author{
Carlos Matos $^{1}$ and Manuel Duarte Ortigueira ${ }^{2}$ \\ ${ }^{1}$ UNINOVA and Escola Superior de Tecnologia, Instituto Politécnico de Setúbal, Portugal \\ cmatos@est.ips.pt, \\ ${ }^{2}$ UNINOVA/DEE Campus da FCT da UNL, Quinta da Torre, 2825-114 \\ Monte da Caparica, Portugal \\ mdo@fct.unl.pt
}

\begin{abstract}
The design and optimization of fractional filters is considered in this paper. Some of the classic filter architectures are presented and their performances relatively to an ideal amplitude spectrum evaluated. The fractional filters are designed using the differential evolution optimization algorithm for computing their parameters. To evaluate the performances of all the filters the quadratic error between the computed amplitude is calculated against an ideal (goal) response. The fractional filters have a better behavior, both in the pass and reject-band.
\end{abstract}

Keywords: Fractional Filters, Fractional Derivative, Optimization.

\section{Introduction}

The importance of filters in signal processing and other engineering areas is unquestionable. Continuous time filters are widely used functional blocks, from simple antialiasing filters preceding ADCs to high-spec channel-select filters in integrated RF transceivers. Four classical classes of filters are currently used: Butterworth, Chebyshev, elliptic and Bessel. Even in the integer order case, filter design is challenging, mainly when the system has to meet a wide set of constrains [1]. Most tools for filter design are based on the transfer functions of the above classes, which impose only requirements related to the magnitude or phase responses.

The design of fractional filters was considered for low orders, [7] and [8]. Here, we will consider the problem with all the generality.

We are dealing essentially with an optimization problem that we will face by using the differential evolution (DE) algorithm [2]. DE is a stochastic, population-based optimization algorithm.

With this algorithm we were able to design fractional filters. To do it we used the poles and zeros as design parameters, together with the fractional order, $\alpha$. This inserts in the problem a new parameter - the fractional derivative order - that gives a new degree of freedom. This may increase the computational burden associated with the optimization algorithm, but leads to more flexible solutions.

The papers outlines as follows. In section 2 we summarize the contribution to technological innovation of this work. In section 3 we describe the main characteristics of 
the filters. The optimization algorithm is described shortly in section 4 after which we present the simulation results. Finally we will present some conclusions.

\section{Contribution to Technological Innovation}

This algorithm opens a way to the design of fractional filters, it allows the calculus and optimization of all the design parameters, including the fractional parameter.

Fractional filters better approximate the ideal response than the classical ones. Their applicability in Industrial Control Systems can be very wide.

The presented results are an incentive in itself to fractional capacitor manufacturers and filter designers, allowing the generalised use of fractional filters and the use of this technology.

\section{Integer Order Filters}

\subsection{Specification of the Filter}

The specifications for a low pass filter are expressed in figure 1. The specifications of the other kinds of filters are similar. So, we will not consider them. Usually we fix the band pass (BP) amplitude equal to 1 . The obtained filter will have amplitude oscillations in the interval

$\left[1-\delta_{\mathrm{p}}, 1+\delta_{\mathrm{p}}\right]$. Other interesting values are:

$\delta_{\mathrm{s}}-$ amplitude of stop band ripple

$\omega_{c}$ - pass band edge frequency

$\omega_{\mathrm{s}}-$ stop band edge frequency

$\Delta \omega_{i}-$ width of the transition band: $\Delta \omega_{i}=\omega_{s}-\omega_{c}$

$\mathrm{A}_{\mathrm{p}}$ - band-pass attenuation

$$
\mathrm{A}_{\mathrm{p}}=20 \log \frac{1+\delta_{p}}{1-\delta_{p}}
$$

$\mathrm{A}_{\mathrm{s}}$ - stop-band attenuation

For other kinds of filters the specifications are similar.

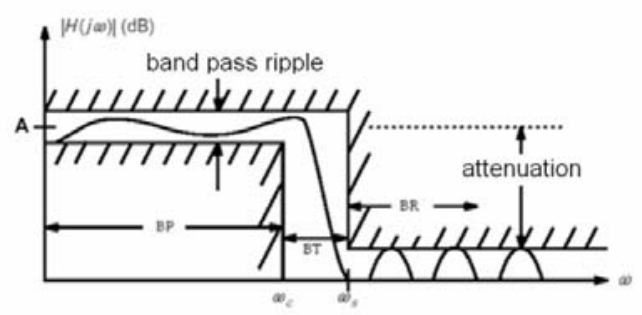

Fig. 1. Specifications of a low pass filter 
In the presented work we considered the filter specifications as described in figure 2 .

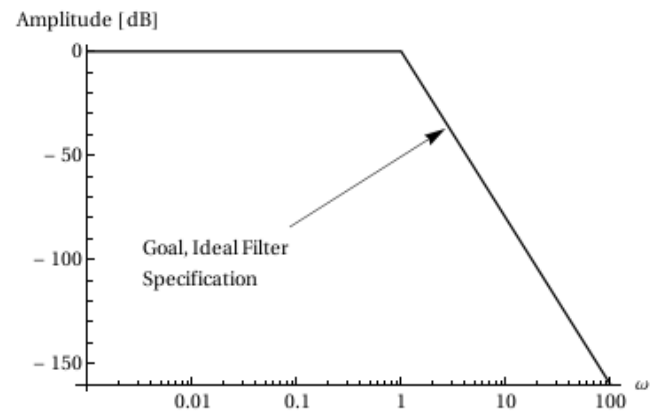

Fig. 2. Specifications of an ideal low pass filter

\section{Simulation Results}

The Genetic Differential Evolution Algorithm was introduced by [5]. It was developed to optimize real parameters of a real valued function. It finds the true global minimum, regardless of the initial parameters, It has fast convergence and it uses a small set of control parameters.

Applying (DE) algorithm to fractional filter design, will lead to filters with minimum quadratic error to a defined goal. The minimization of the quadratic error of the frequency response, supply us with the values of the zeroes, poles and fractional order $\alpha$.

\section{$3^{\text {rd }}$ Order Integer Low-Pass Filter}

Suppose that we want to approximate the ideal low pass filter defined by:

$$
\begin{cases}1 & \text { if } 0 \leq \omega \leq 1 \\ -20 \log \left(\omega^{3}\right) & \text { if } \omega>1\end{cases}
$$

With the usual $3^{\text {rd }}$ order Low-Pass filters, Butterworth, Bessel, Elliptic and Chebyshev. The quadratic error of these filters compared to the ideal response, for $\omega \in] 0,10]$ is: Butterworth: 1.90658, Bessel: 18.4252, Elliptic: 5649.03, Chebyshev: 282.28

\section{$3^{\text {rd }}$ Order Fractional Filter}

We define the fractional Butterworth filter, $\alpha$ the fractional parameter, as:

$$
H(s)=\frac{1}{s^{3 \alpha}+2 s^{2 \alpha}+2 s^{\alpha}+1}
$$


First we will use a $3^{\text {rd }}$ order all-pole fractional filter (2). We impose that we have two complex conjugate poles and a real pole. The optimization algorithm is intended to calculate the poles and $\alpha$ that minimize the quadratic error using the genetic differential evolution algorithm.

For the above defined ideal filter approximation, we obtained, Pole $1=$ $0.386955 \pm 0.890131$, Pole $2=-1.13249, \alpha=1.00031$ and a quadratic error: 0.331989

The fractional transfer function is given by:

$$
\mathrm{H}(\mathrm{s})=\frac{1}{\mathrm{~s}^{3.00094}+1.9064 \mathrm{~s}^{2.00063}+1.818 \mathrm{~s}^{1.00031}+1.06688}
$$

The responses are depicted in figure 3 for the amplitude and figure 4 for the phase.

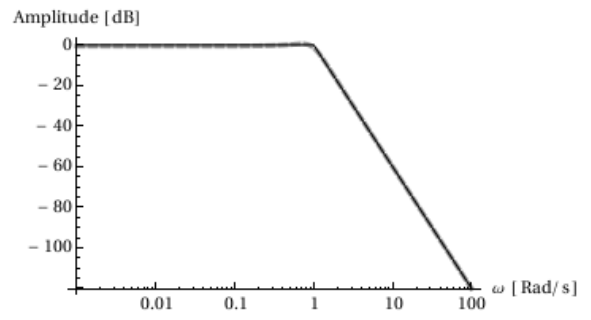

Fig. 3. Amplitude response of $3^{\text {rd }}$ order fractional filter. Goal in solid Black and designed in dashed gray.

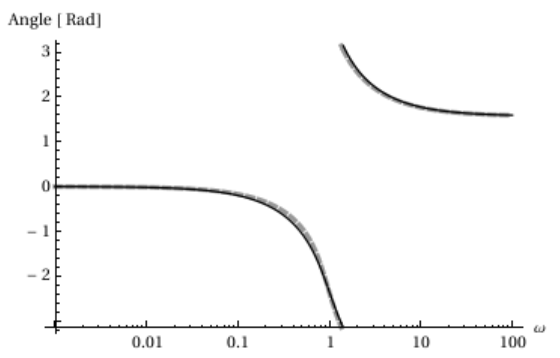

Fig. 4. Phase response of 3 rd order fractional filter (dashed gray) and $3^{\text {rd }}$ order LP Butterworth (solid black)

The parameter $\alpha$ is very close to 1 as the filter, a $3^{\text {rd }}$ order is very close to the integer response given by the Butterworth filter.

Now we are going to use the same $3^{\text {rd }}$ order fractional filter to approximate an ideal $4^{\text {th }}$ order Low-Pass filter defined by:

$$
\begin{cases}1 & \text { if } 0 \leq \omega \leq 1 \\ -20 \log \left(\omega^{4}\right) & \text { if } \omega>1\end{cases}
$$

Proceeding as above we calculate $\alpha$ and the poles:

Pole $1=-0.0000449527+\mathrm{j} 0.817145$, Pole $2=-1.67915, \alpha=1.34776$ with a quadratic error of: 24.6592 . And the transfer function is:

$$
H(s)=\frac{1}{s^{4.043}+1.679 s^{2.696}+.668 s^{1.348}+1.121}
$$

This is a case where $\alpha>1$, because the ideal filter decay is bigger than the integer Butterworth capacity. The responses are depicted in figure 5 for the amplitude and figure 6 for the phase. 


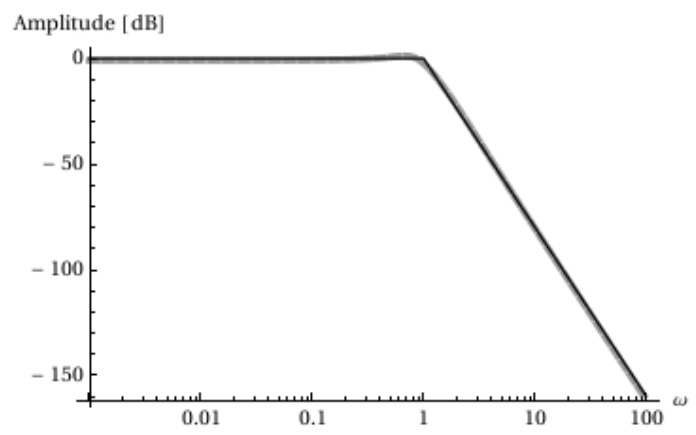

Fig. 5. Amplitudes of fractional 3rd order fractional low pass filter (dashed grey) and ideal 4th order LP response (solid black)

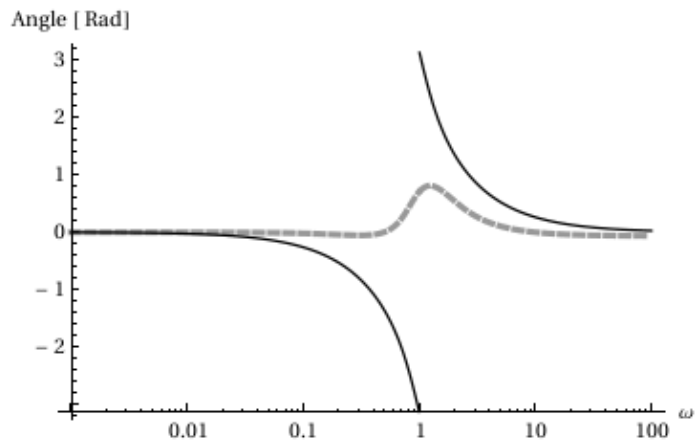

Fig. 6. Phase response of 3rd order fractional Low Pass filter (dashed grey) compared with Butterworth (solid black)

\section{Conclusions}

We just considered the design of fractional filters and compared their performances with similar classic filters, from the point of view of the amplitude spectrum. The fractional filters approximate much better the ideal filter than the classic ones.

One rule of design should be starting by the use of the fractional filter derived from the integer that better approximates the goal. In our study, we used Butterworth filters as the starting point. We demonstrated that if the integer filter order is smaller than the needed response $\alpha$ will be bigger than one, on the other hand, if we start with an integer filter that has an order higher than needed then $\alpha$ will be smaller than one.

We have shown that the fractional filters outperform the integer ones. By changing poles location and finding a suitable $\alpha$ a fractional filter can perform as a higher or lower order integer filter with advantages. One of the main advantages is that we can have a smaller quadratic error between the goal ideal filter and the designed filter. Another important advantage is that a $\mathrm{N}$ order fractional filter with $\alpha>1$ performs as 
an integer filter of order $>\mathrm{N}$, so we can have a filter of order $>\mathrm{N}$ but with the complexity of a $\mathrm{N}$ order.

The same fractional approach will be applied to other type of classical filters and the results will be presented in future work.

\section{References}

1. Damera-Venkata, N., Evans, B.L.: An automated framework for multicriteria optimization of analog filter designs. IEEE Transactions on Circuits and Systems - II: Analog and Digital Signal Processing 46(8), 981-990 (1999)

2. Haupt, R.L., Haupt, S.E.: Practical Genetic Algorithms, 2nd edn. John Wiley \& Sons, Inc., New Jersey (2004)

3. Nedelea, L., Topa, M., Neag, M.: Computer-Aided Network Function Approximation for Multicriteria Filter Design. In: SCS 2003 - International Symposium on Signals, Circuits and Systems, Iaşi, Romania, pp. 81-84 (2003)

4. Ortigueira, M.D.: A coherent approach to non integer order derivatives. Signal Processing Special Section: Fractional Calculus Applications in Signals and Systems 10, 2505-2515 (2006)

5. Ortigueira, M.D.: Introduction to Fractional Signal Processing. Part 2: Discrete-Time Systems. In: IEE Proc. On Vision, Image and Signal Processing, vol. (1), pp. $71-78$ (2000)

6. Price, K.V., Storn, R.M., Lampinene, J.A.: Differential Evolution: A Practical Approach to Global Optimization. Springer, Berlin (2005)

7. Radwan, A.G., Soliman, A.M., Elwakil, A.S.: First Order Filters Generalized to the Fractional Domain. Journal of Circuits, Systems and Computers 17(1), 55-66 (2008)

8. Radwan, A.G., Elwakil, A.S., Soliman, A.M.: On the Generalizations of Second-Order Filters to the Fractional-Order Domain (2009) 